\title{
Unilateral Phrenic Nerve Plasy: A Rare Manifestation of Vincristine Neurotoxicity: Correspondence
}

\author{
Kunihiko Moriya • Yosuke Kakisaka • Masaei Onuma • \\ Yoji Sasahara $\cdot$ Shigeo Kure
}

Received: 28 September 2013 / Accepted: 26 February 2014 /Published online: 22 March 2014

(C) Dr. K C Chaudhuri Foundation 2014

To the Editor: We read with interest the article written by Dhingra et al. entitled "Unilateral phrenic nerve palsy: A rare manifestation of vincristine neurotoxicity" [1]. Here we present a patient with mesenchymal chondrosarcoma (MC) who developed hiccups repeatedly after receiving vincristine infusion. In addition to our case, several agents such as corticosteroid and cyclophosphamide infusion are also known to cause drug-induced hiccups [2-4].

A 9-y-old boy presented with pain and swelling in the right knee for 1 mo. Limb-sparing operation with wide excision of the tumor and pasteurized autologous bone graft was performed. He was given six courses of adjuvant chemotherapy, of alternative course of combination of vincristine, doxorubicin, cyclophosphamide (VDC), combination of ifosfamide, etoposide (IE), every $3 \mathrm{wk}$ intervals. When he received $1.5 \mathrm{mg} / \mathrm{m}^{2}$ of vincristine and granisetron as an anti-emesis, intravenously on the first day of the VDC regimen, he developed hiccups for half an hour after vincristine infusion. The event occurred reproducibly, never occurred at IE regimen, when the patient also received granisetron. The hiccups improved spontaneously later without any treatment.

The temporal profile of hiccup disappearance, $6 \mathrm{~h}$ after vincristine administration spontaneously, might be explained exponential regression curve of blood concentration of vincristine, although the mechanism still remains unknown [5].

Impaired peripheral nerve functions and bowel movement are well known adverse effects caused by vincristine. Vincristine could cause hiccups as a part of combination chemotherapy.

Conflict of Interest None.

Role of Funding Source None.

\section{References}

1. Dhingra D, Sethi GR, Mantan M. Unilateral phrenic nerve palsy: A rare manifestation of vincristine neurotoxicity. Indian J Pediatr. 2013. doi:10.1007/s12098-013-1116-6.

2. Ifran A, Kaptan K, Beyan C. Intractable hiccups may develop with cyclophosphamide infusion. Am J Hematol. 2004;77:319-20.

3. Mark A. Diagnosis and management of hiccups in the patient with advanced cancer. J Support Oncol. 2009;7:122-30.

4. Cersosimo RJ, Brophy MT. Hiccups with high dose dexamethasone administration: A case report. Cancer. 1998;82:412-4.

5. Nelson R. The comparative clinical pharmacology and pharmacokinetics of vindesine, vincristine, and vinblastine in human patients with cancer. Med Pediatr Oncol. 1982;10:115-27. 